\title{
Tratamento da lesão em onda do quadril através da microfratura reversa: Estudo clínico retrospectivo
}

\section{Treatment of Hip Wave Lesion Using Reverse Microfracture: A Retrospective Clinical Study}

\author{
Leandro Calil De Lazari1 ${ }^{10}$ \\ Fernando Jorge Garcia ${ }^{3}$ \\ ${ }^{1}$ Serviço Residência de Ortopedia do Grupo São Lucas, Hospital Care,
Ribeirão Preto, SP, Brasil
2 Grupo do Quadril, Hospital das Clínicas, Faculdade de Medicina de Ribeirão
Preto, Universidade de São Paulo (FMRP - USP), Ribeirão Preto, SP, Brasil
${ }^{3}$ Hospital Estadual de Sumaré, Universidade de Campinas (Unicamp),
Campinas, SP, Brasil,
}

Celso Herminio Ferraz Picado ${ }^{2}$ Flavio Luis Garcia ${ }^{20}$

Endereço para correspondência Leandro Calil De Lazari, PhD, Av Itatiaia 1049, Higienópolis, Ribeirão Preto, SP, 14025-070, Brasil (e-mail: calil.delazari@gmail.com; contato@leandrocalil.com.br).

Rev Bras Ortop 2022;57(1):144-149.

\section{Resumo \\ Palavras-chave \\ - artroplastia de quadril \\ - artroscopia \\ - microfratura \\ - impacto femoroacetabular}

Objetivo Avaliar o tratamento desta lesão, através da microfratura reversa, que é um procedimento simples e sem aumento de insumos na cirurgia.

Métodos Foram analisados retrospectivamente 19 pacientes submetidos a tratamento da lesão em onda no acetábulo, através da microfratura reversa. Utilizamos a ressonância nuclear magnética (RNM) no momento do diagnóstico e 6 meses após a cirurgia, avaliação funcional pelo Harris Hip Score (HHS) e escala visual e analógica (EVA) da dor no pré-operatório, e 3 e 6 meses após a cirurgia.

Resultado os dados estatísticos mostraram melhora significativa do HHS e EVA da dor após 6 meses da cirurgia. A RNM após 6 meses da cirurgia mostrou que na área que foi submetida à microfratura reversa, a cartilagem se apresentou com as mesmas características visuais que nas áreas sem lesão condral.

Conclusão Concluímos que a microfratura reversa se mostrou eficaz e reprodutível no tratamento da lesão em onda.

Objective The present study aims to evaluate the treatment of hip wave lesion using reverse microfracture, which is a simple and cheap surgical procedure.

Methods We retrospectively analyzed 19 patients with acetabular wave lesion treated with reverse microfracture. The patients were assessed by magnetic nuclear resonance imaging (MRI) at the time of diagnosis and 6 months after the surgery and functionally evaluated using the Harris Hip Score (HHS) and the visual analogue scale (VAS) for pain in the preoperative period, and 3 and 6 months after the surgery.

Trabalho desenvolvido no Grupo São Lucas, Hospital Care,

Ribeirão Preto, SP, Brasil.

recebido

09 de Abril de 2020

aceito

02 de Outubro de 2020

Publicado on-line

Abril 19, 2021
DOI https://doi.org/

$10.1055 / \mathrm{s}-0040-1722259$

ISSN 0102-3616.
(C) 2021. Sociedade Brasileira de Ortopedia e Traumatologia. All rights reserved.

This is an open access article published by Thieme under the terms of the Creative Commons Attribution-NonDerivative-NonCommercial-License, permitting copying and reproduction so long as the original work is given appropriate credit. Contents may not be used for commercial purposes, or adapted, remixed, transformed or built upon. (https://creativecommons.org/ licenses/by-nc-nd/4.0/)

Thieme Revinter Publicações Ltda., Rua do Matoso 170, Rio de Janeiro, RJ, CEP 20270-135, Brazil 
Results The statistical data showed a significant improvement in HHS and VAS 6 months after the surgery. Six months after the surgery, the MRI revealed that the areasubjected to reverse microfracture presented cartilage with the same visual characteristics observed in areas with no chondral injury.

Conclusion We conclude that the reverse microfracture proved to be an effective, reproducible method for the treatment of wave lesion.

\section{Introdução}

A lesão em onda representa uma pré-delaminação ou delaminação profunda da cartilagem articular ou do complexo condrolabral sem extensão intraarticular. ${ }^{1}$ Este tipo de lesão é de difícil diagnóstico pré-operatório, sendo reconhecido, geralmente, durante a cirurgia. ${ }^{1}$ Como se trata de uma lesão da cartilagem articular pode causar graves problemas na articulação do quadril, como artralgia, sinovite, formação de corpos livres e/ou evolução para osteoartrite. ${ }^{2}$ O desafio para os cirurgiões é a indicação cirúrgica correta associada ao tratamento clínico destinado a curar a lesão.

Uma opção para o tratamento das lesões em onda é a técnica artroscópica descrita como microfratura reversa. ${ }^{3}$ Esta técnica é realizada com auxílio de um portal anterolateral acessório proximal, no qual se consegue a angulação e posição adequadas para microfraturas do osso acetabular sem causar lesões da cartilagem condrolabral, reduzindo assim a pressão das bolhas formadas pela lesão em onda e induzindo a formação de um coágulo que, associado à reação cicatricial óssea, pode atuar como um adesivo natural que une a cartilagem ao osso. ${ }^{3}$

O presente estudo tem como objetivo avaliar os resultados clínicos e radiológicos de 19 pacientes portadores de lesão tipo onda, tratados com a técnica de microfratura reversa.

\section{Material e Métodos}

Estudo realizado após a aprovação deste estudo no Conselho de Ética e Pesquisa (CEP) do grupo dos hospitais São Lucas e Ribeirânia, Ribeirão Preto - SP, a pesquisa foi desenvolvida conforme preconiza a Resolução $\mathrm{n}^{\circ} 466 / 12$ do Conselho Nacional de Saúde (CNS).
Trata-se de um estudo retrospectivo, descritivo e quantitativo, por meio de dados coletados após análise e revisão de prontuários de pacientes que apresentavam lesão em onda e foram tratados com a técnica de microfratura reversa no período de maio de 2016 a outubro de 2017.

A técnica artroscópica descrita como microfratura reversa, que foi usada no presente estudo, é realizada com posicionamento do paciente em uma mesa de tração, em decúbito dorsal horizontal. ${ }^{3} \mathrm{Com}$ auxílio de radioscopia, o membro inferior afetado é tracionado até obter-se um espaço articular de 2 centímetros. Após a montagem dos campos, são feitos os portais anterolateral e anterior para visualização do compartimento intra-articular e diagnóstico da lesão em onda na cartilagem acetabular. Para tratamento da lesão, é criado um portal anterolateral acessório proximal (ACP) com uma angulação de 30 a $45^{\circ}$ proximal e anterior ao portal anterolateral e a meio caminho da espinha ilíaca anterosuperior e uma linha perpendicular ao topo do trocânter maior. ${ }^{3}$

Por meio deste portal (ACP), é realizada a técnica da microfratura reversa sob visão direta com uma broca de baixa rotação, podendo-se realizar quantas microfraturas forem necessárias para tratamento da lesão, com uma distância de 3 a 4 mm entre elas ${ }^{4}$ (-Figura $\mathbf{1}$ ).

Foram selecionados e incluídos 19 prontuários de um total de 28 pacientes operados, dentre os quais tiveram os seguintes dados avaliados: gênero, idade, lateralidade, data da cirurgia, tipo de impacto femoroacetabular (IFA), ressonância nuclear magnética (RNM) no momento do diagnóstico e seis meses após a cirurgia, avaliação funcional pelo Harris Hip Score (HHS) e escala visual e analógica (EVA) da dor no pré-operatório, com 3 e 6 meses de cirurgia. Na EVA foi considerado como dor leve a graduação de 0 a 2 , moderada de 3 a 7 e intensa de 8 a 9 (-Tabela $\mathbf{1}$ ).

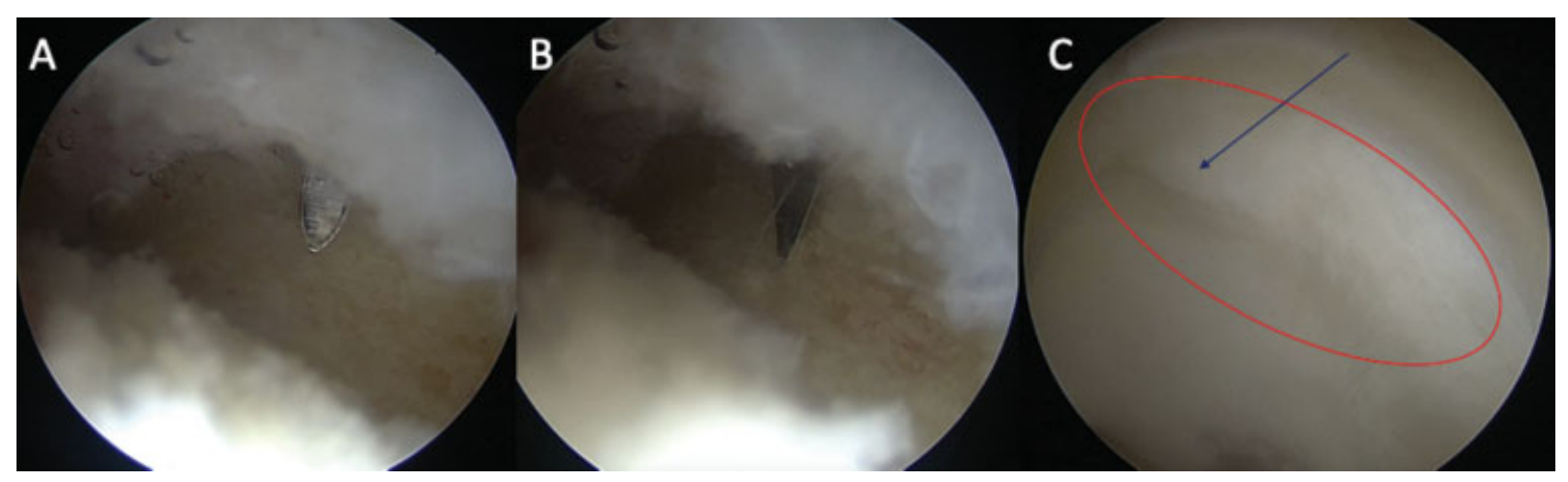

Fig. 1 (A) imagem mostrando o introdutor canulado e a posição desejada para a introdução da broca de 2,3 mm. (B) imagem mostrando a broca, e o início da perfuração. (C) imagem da lesão em onda (elipse vermelha) e a perfuração do osso subcondral, sem romper a cartilagem (seta). 


\begin{tabular}{|c|c|c|c|c|c|c|c|c|c|c|c|c|c|c|c|c|c|c|c|}
\hline 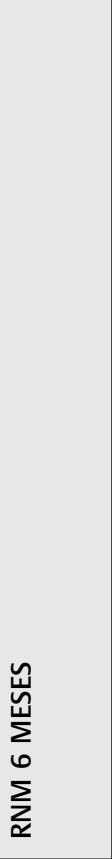 & 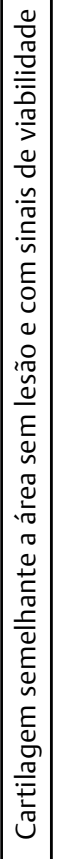 & 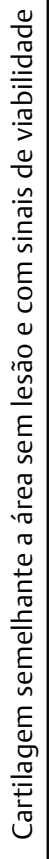 & 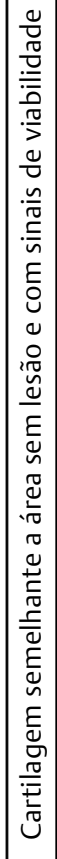 & 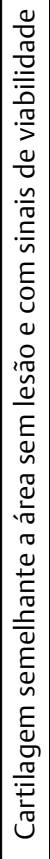 & 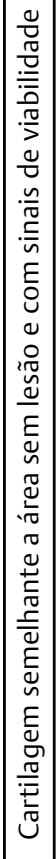 & 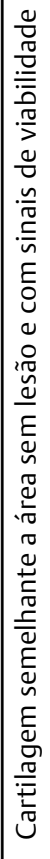 & 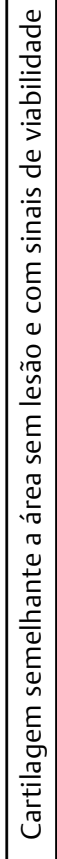 & 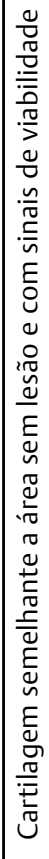 & 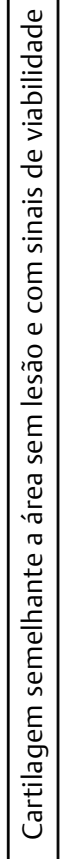 & 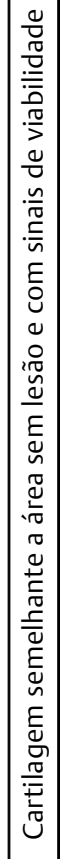 & 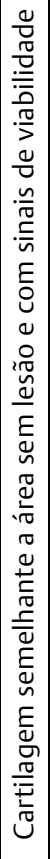 & 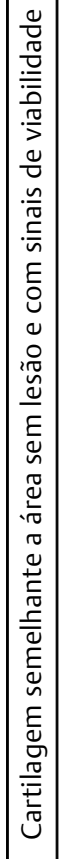 & 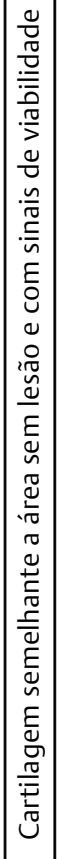 & 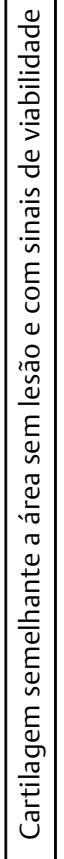 & 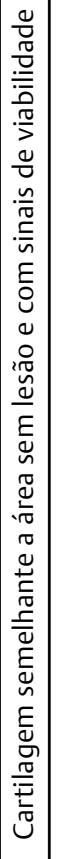 & 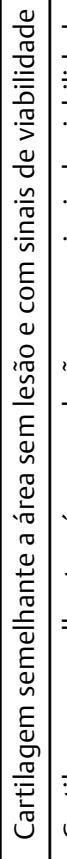 & 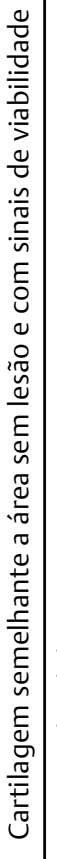 & 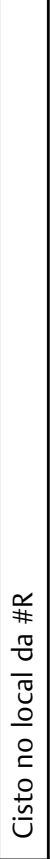 & 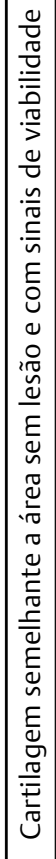 \\
\hline$\sum \sum_{0}^{\breve{u ̈}}$ & 0 & $m$ & 0 & - & $\sim$ & - & 0 & - & $\sim$ & 0 & - & - & - & $\sim$ & 0 & - & $\sim$ & $m$ & 0 \\
\hline 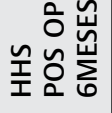 & $\stackrel{\circ}{\circ}$ & $\bar{\infty}$ & Һ & $\bar{\sigma}$ & 曲 & б & $\stackrel{\circ}{\circ}$ & $\stackrel{9}{1}$ & ৪ & : & nू & @ & б & б & 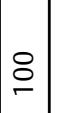 & б & б। & श & 응 \\
\hline m出 & $m$ & in & $m$ & $\nabla$ & 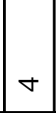 & $v$ & - & $\sigma$ & in & $\sim$ & in & $m$ & $m$ & $\nabla$ & - & $m$ & $m$ & $\nabla$ & - \\
\hline 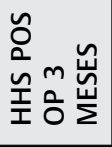 & $\infty$ & $ং$ & $\stackrel{\llcorner}{\infty}$ & $\stackrel{\llcorner}{\infty}$ & $\stackrel{\llcorner}{\wedge}$ & $\begin{array}{l}\infty \\
\infty\end{array}$ & ৪ & $\stackrel{R}{\wedge}$ & $\stackrel{n}{\wedge}$ & б & б & $\stackrel{2}{\wedge}$ & I & $\stackrel{\llcorner}{\wedge}$ & б. & $\infty$ & $\stackrel{2}{\wedge}$ & $\stackrel{\text { № }}{\wedge}$ & $\bar{\sigma}$ \\
\hline 㐍容。 & $\wedge$ & $\infty$ & $\wedge$ & $\wedge$ & 6 & 6 & $\nabla$ & $\sigma$ & $\infty$ & 6 & $r$ & $\wedge$ & $\infty$ & $\infty$ & $\wedge$ & $\wedge$ & $\infty$ & $\infty$ & in \\
\hline 愛営。 & 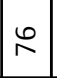 & 亡 & $\stackrel{\sim}{*}$ & 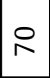 & 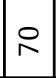 & $\stackrel{\sim}{\star}$ & $\bar{\infty}$ & เి & $\stackrel{\infty}{\infty}$ & $\bar{\infty}$ & 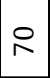 & 5 & i̊n & 边 & $\begin{array}{l}\infty \\
\infty\end{array}$ & $\stackrel{\circ}{1}$ & $\bar{\sigma}$ & in & $\bar{\infty}$ \\
\hline 올 & $\frac{0}{\Sigma}$ & 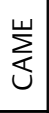 & 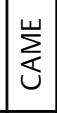 & $\frac{0}{\Sigma}$ & $\frac{0}{\Sigma}$ & $\frac{0}{\Sigma}$ & 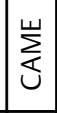 & $\sum_{ভ}^{\mathrm{U}}$ & 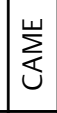 & $\frac{\circ}{\bar{n}}$ & $\frac{\circ}{\Sigma}$ & $\frac{O}{\frac{0}{\Sigma}}$ & $\sum^{\mathrm{U}}$ & $\sum^{\mathrm{U}}$ & $\sum^{\mathrm{U}}$ & $\begin{array}{l} \\
\frac{2}{\Sigma} \\
\end{array}$ & 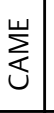 & $\frac{0}{\frac{0}{\Sigma}}$ & $\frac{0}{\Sigma}$ \\
\hline 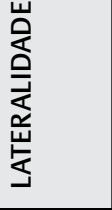 & 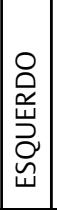 & $\begin{array}{l}\text { 은 } \\
\underline{\underline{\underline{\alpha}}} \\
\underline{\bar{\alpha}}\end{array}$ & 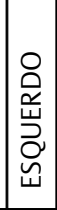 & $\begin{array}{l}\text { 을 } \\
\text { 岃 } \\
\text { 言 }\end{array}$ & 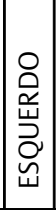 & 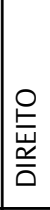 & \begin{tabular}{|l} 
을 \\
产 \\
立
\end{tabular} & 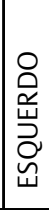 & $\begin{array}{l}\text { 음 } \\
\text { 岕 } \\
\text { 立 }\end{array}$ & $\begin{array}{l}\text { 을 } \\
\text { 产 } \\
\underline{\overline{0}}\end{array}$ & $\begin{array}{l}\text { 을 } \\
\text { 荘 }\end{array}$ & $\begin{array}{l}\text { 음 } \\
\text { 产 } \\
\text { 咅 }\end{array}$ & 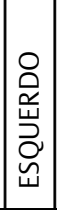 & 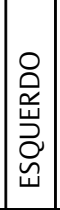 & $\begin{array}{l}\stackrel{0}{\mathrm{~W}} \\
\underline{\underline{\underline{\alpha}}} \\
\end{array}$ & 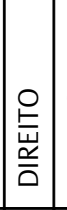 & $\begin{array}{l}\text { 을 } \\
\stackrel{\underline{\underline{w}}}{\bar{\Delta}}\end{array}$ & & 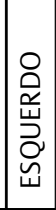 \\
\hline 总 & 号 & 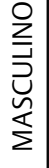 & 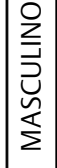 & 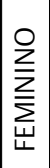 & 足 & 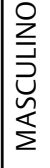 & 足 & $\sum_{\text {出 }}^{\stackrel{O}{\sum}}$ & 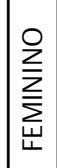 & 足 & 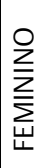 & $\sum_{\text {出 }}^{\stackrel{O}{\sum}}$ & 呈 & $\sum_{\text {出 }}^{\stackrel{0}{z}}$ & 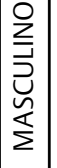 & 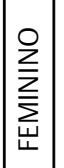 & $\sum_{\text {出 }}^{\stackrel{O}{\gtrless}}$ & & 足 \\
\hline 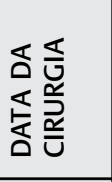 & 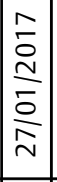 & \begin{tabular}{|l}
0 \\
0 \\
$\frac{1}{2}$ \\
$\frac{2}{2}$ \\
0 \\
\end{tabular} & $\begin{array}{l}\frac{\hat{\sigma}}{2} \\
\frac{N}{m} \\
\frac{0}{0} \\
0\end{array}$ & 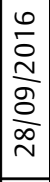 & \begin{tabular}{|l|}
0 \\
0 \\
$\frac{1}{2}$ \\
$\frac{1}{2}$ \\
$\frac{2}{8}$ \\
\end{tabular} & $\mid \begin{array}{l}0 \\
\frac{1}{1} \\
\frac{1}{8} \\
\frac{0}{0} \\
0 \\
\end{array}$ & 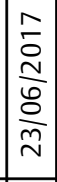 & $\begin{array}{l}\frac{0}{2} \\
\frac{1}{d} \\
\frac{\partial}{0} \\
\frac{0}{m}\end{array}$ & $\begin{array}{l}\frac{1}{2} \\
\stackrel{N}{a} \\
\frac{0}{0} \\
\\
\end{array}$ & 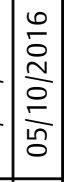 & $\begin{array}{l}\frac{1}{0} \\
\frac{1}{0} \\
\frac{1}{2} \\
\text { a }\end{array}$ & $\frac{\hat{D}}{\frac{1}{N}}$ & $\begin{array}{l}0 \\
0 \\
\frac{1}{2} \\
\frac{2}{8} \\
0 \\
\end{array}$ & $\begin{array}{l}\frac{1}{2} \\
\frac{1}{2} \\
\frac{2}{2} \\
\stackrel{2}{2} \\
\end{array}$ & \begin{tabular}{|c|}
$\frac{1}{2}$ \\
$\frac{1}{\infty}$ \\
0 \\
$\frac{1}{2}$ \\
0
\end{tabular} & $\begin{array}{l}0 \\
0 \\
\frac{1}{\infty} \\
0 \\
\frac{0}{9} \\
\frac{1}{2}\end{array}$ & $\begin{array}{l}\frac{0}{0} \\
\frac{N}{0} \\
\frac{D}{n} \\
\stackrel{N}{N}\end{array}$ & 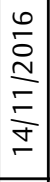 & 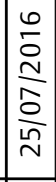 \\
\hline & - & $\sim$ & $m$ & $\nabla$ & in & 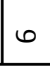 & $\wedge$ & 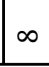 & $a$ & 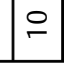 & 1 & $\simeq$ & -1 & \pm & $\stackrel{\llcorner}{\llcorner}$ & $\stackrel{\circ}{\circ}$ & $\therefore$ & $\stackrel{\infty}{\leftarrow}$ & 9 \\
\hline
\end{tabular}


Nove pacientes foram excluídos do presente estudo devido à falta de documentação necessária ou perda de seguimento.

Os critérios de inclusão foram: IFA tipo misto ou CAM, lesão em onda isolada ou associada a lesão labral, fisioterapia assistida, ${ }^{5}$ pacientes operados pelo mesmo cirurgião. E os critérios de exclusão foram: falta do HHS ou RNM no pré ou pós-operatório, lesão condral tipo clivagem, delaminação ou exposição do osso subcondral associada a lesão em onda, displasia acetabular, quadril encarcerado ou sobrecobertura excessiva, coxa profunda ou protrusa, revisão de artroscopia, instabilidade coxofemoral, hiper mobilidade articular seguindo os critérios de Beighton. ${ }^{6}$

\section{Análise estatística}

Os dados foram avaliados por meio de uma estatística descritiva, em que foi possível a caracterização da amostra quanto às variáveis coletadas.

Para verificar a associação entre as variáveis qualitativas, os dados foram submetidos ao Teste Exato de Fisher. ${ }^{7}$

Foram utilizados testes t de Student, no qual consiste em comparar duas médias provenientes de amostras não pareadas. Para a utilização deste teste é necessário testar se as variâncias dos dois grupos são estatisticamente iguais, e se os dados seguem distribuição normal.

Todas as análises estatísticas foram realizadas com a utilização do software estatístico SAS. Valores de p menores que 0,05 foram considerados significativos.

$\mathrm{Na}$ análise estatística do Harris Hip Scores em relação ao tempo, foram classificados os resultados em pobre quando apresenta menos de 70 pontos, razoável quando apresenta entre 70 e 80 pontos, bom quando apresenta entre 80 e 90 pontos e excelente quando for maior que 90 pontos. $^{8}$

\section{Resultados}

Dos 19 pacientes avaliados, 15 (78,95\%) são do sexo feminino, enquanto quatro $(21,05 \%)$ do sexo masculino. Onze $(57,89 \%)$

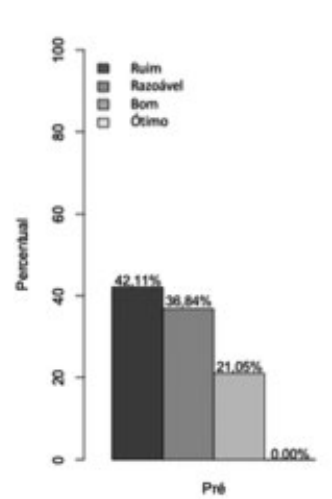

Harris Hip Score
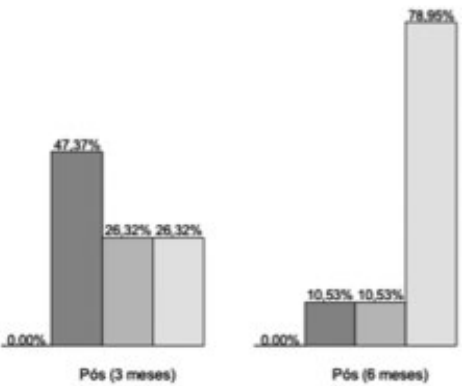

Fig. 2 Gráfico mostrando a melhora dos resultados pré-operatório e pós operatório segundo o Harris Hip Score.

pacientes operaram o lado direito e oito $(42,11 \%)$ o lado esquerdo.

Em nove (47,37\%) pacientes foram visualizadas IFA tipo CAM e os outros $10(52,63 \%)$ do tipo misto. Três (75\%) dos homens e 6 (40\%) das mulheres apresentavam IFA tipo CAM. Nenhum paciente apresentou IFA tipo pincer exclusiva.

Somente um paciente $(5,26 \%)$ apresentou complicação pós-operatória, com formação de cisto na região da microfratura.

Na avaliação estatística temporal, o HHS demonstra no pré-operatório um percentual de $42,11 \%$ dos pacientes com escore pobre, 36,84\% razoável e 21,05\% bom. Após 3 meses de cirurgia, $47,37 \%$ dos pacientes apresentavam escore razoável, $26,32 \%$ bom e 26,32\% excelente. Após 6 meses de cirurgia, $10,53 \%$ dos pacientes apresentaram resultado razoável, $10,53 \%$ bom e 78,95\% excelente $(p<0,05)$ ( - Tabelas 2 e 3 e - Figura 2).

Em relação à avaliação temporal da EVA da dor, 63,16\% dos pacientes apresentavam dor moderada e $36,84 \%$ dor intensa no pré-operatório. Após 3 meses da cirurgia, 26,32\% apresentavam dor leve e 73,68\% dor moderada, e após 6 meses da

Tabela 2 Média do Harris Hip Score antes da cirurgia (T0), e com 3 meses (T3) e 6 meses de pós operatório (T6)

\begin{tabular}{|l|l|l|l|l|l|l|}
\hline HHS X Tempo & N & Média & Desvio padrão & Mínimo & Mediana & Máxima \\
\hline Tempo & 19 & 67.53 & 11.73 & 41.00 & 70.00 & 86.00 \\
\hline T0 & 19 & 81.89 & 7.52 & 70.00 & 85.00 & 91.00 \\
\hline T3 & 19 & 92.11 & 7.15 & 79.00 & 91.00 & 100.00 \\
\hline T6 &
\end{tabular}

Tabela 3 Mostra que houve diferença significativa entre o pré-operatório e o pós-operatório (T3 e T6) com relação ao Harris Hip Score, usando um valor de $p$ de $5 \%$

\begin{tabular}{|l|l|l|l|l|}
\hline \multicolumn{2}{|l|}{ Média HHS X Tempo } & Estimativa & Valor de $\boldsymbol{p}^{*}$ & \multicolumn{3}{l|}{ Intervalo de confiança de 95\% } \\
\hline Comparação & -14.3684211 & $<0.0001$ & -20.2495574 & -8.4872847 \\
\hline (T0-T3) & -24.5789474 & $<0.0001$ & -30.4600837 & -18.6978111 \\
\hline (T0-T6) & -10.2105263 & 0.0010 & -16.0916626 & -4.3293900 \\
\hline (T3-T6) &
\end{tabular}


148 Tratamento da lesão em onda do quadril através da microfratura reversa De Lazari et al.

Tabela 4 Análise da escala visual analógica (EVA) da dor através do método de Fisher e com o valor de $p$ de $5 \%$.

\begin{tabular}{|l|l|l|l|l|}
\hline \multicolumn{4}{|l|}{ Tempo X EVA } & \multirow{2}{*}{ Total } \\
\hline \multirow{2}{*}{ Tempo } & \multicolumn{1}{|l|}{ EVA $^{*}$} & 3 & \\
\cline { 2 - 4 } & $\mathbf{1}$ & $\mathbf{2}$ & $\mathbf{3}$ & \\
\hline T0 & 0 & 12 & 7 & 19 \\
& 0.00 & 61.16 & 36.84 & 100.00 \\
\hline T3 & 5 & 14 & 0 & 19 \\
& 26.32 & 73.68 & 0.00 & 100.00 \\
\hline T6 & 17 & 2 & 0 & 19 \\
& 89.47 & 10.53 & 0.00 & 10.00 \\
\hline Total & 22 & 28 & 7 & 57 \\
\hline
\end{tabular}

*EVA 1 representa dor leve, 2 moderada e 3 intensa.

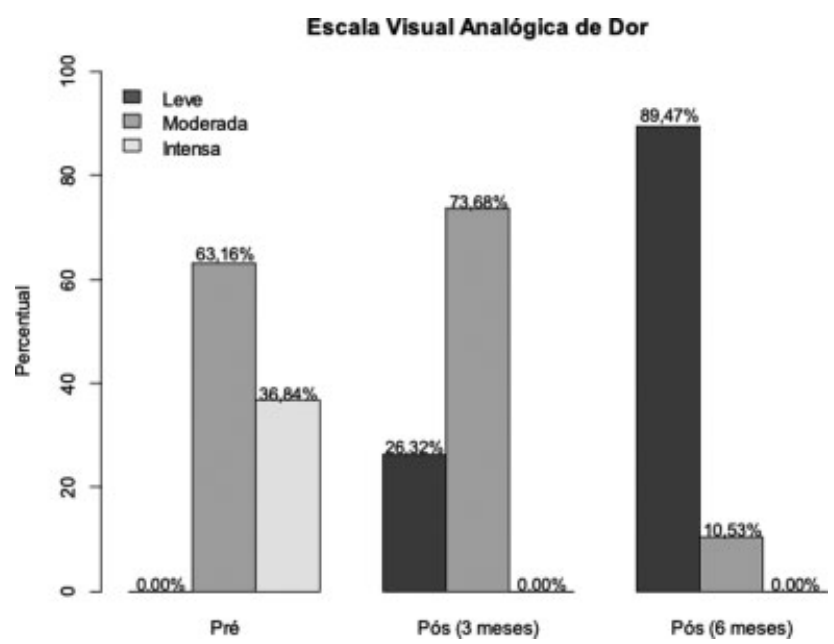

Fig. 3 Gráfico ilustrando a diminuição escalonada da dor segunda a escala visual analógica entre os períodos pré-operatório, $3^{\circ}$ e $6^{\circ}$ mês. cirurgia, $89,47 \%$ apresentavam dor leve e $10,53 \%$ dor moderada $(p<0,05)$. Os dados mostram a melhora significa da dor segundo a tabela EVA após 3 e 6 meses da cirurgia ( - Tabela 4 e - Figura 3).

Foi realizada uma RNM após 6 meses da cirurgia em todos os pacientes, que demonstrou que a cartilagem tratada apresentava intensidade de sinal semelhante à cartilagem normal adjacente em 18 pacientes (- Figura 4). Um paciente apresentou a formação de um cisto na área das microfraturas (-Tabela 1).

\section{Discussão}

Técnicas de microfraturas, ${ }^{4,9}$ reparo direto, ${ }^{4,10}$ reparo com adesivos de fibrina, ${ }^{11,12}$ transplante de condrócitos autólogos (ACT), ${ }^{13}$ condrôgenese induzida por matriz extracelular (AMIC, na sigla em inglês), ${ }^{14}$ mosaicoplastia, ${ }^{15}$ transplante de aloenxerto osteocondral ${ }^{2}$ e implantação de células tronco na matriz ${ }^{2}$ são técnicas que apresentaram bons resultados em algumas lesões específicas e resultados insatisfatórios em outras.

O presente estudo relata os resultados de um procedimento artroscópico indicado para lesões em onda da cartilagem acetabular. Como se trata de um procedimento novo, poucos dados foram encontrados na literatura para realizar comparações. $^{12}$

Dentre as lesões observadas, a maioria (52,63\%) dos pacientes apresentavam lesão tipo mista, e quando se avalia as lesões em pacientes do sexo masculino, 3 (75\%) eram do tipo CAM, o que deixa de acordo com a literatura atual, ${ }^{1}$ a qual demonstra o tipo misto como o mais comum e o came isolado mais frequente em homens. ${ }^{1}$

O que apresenta concordância com a literatura é que o HHS e EVA da dor apresentaram uma melhora progressiva dos sintomas quando comparadas no pré-operatório, com três meses e após seis meses da cirurgia. Na RNM préoperatória observamos a região condral lesionada mais

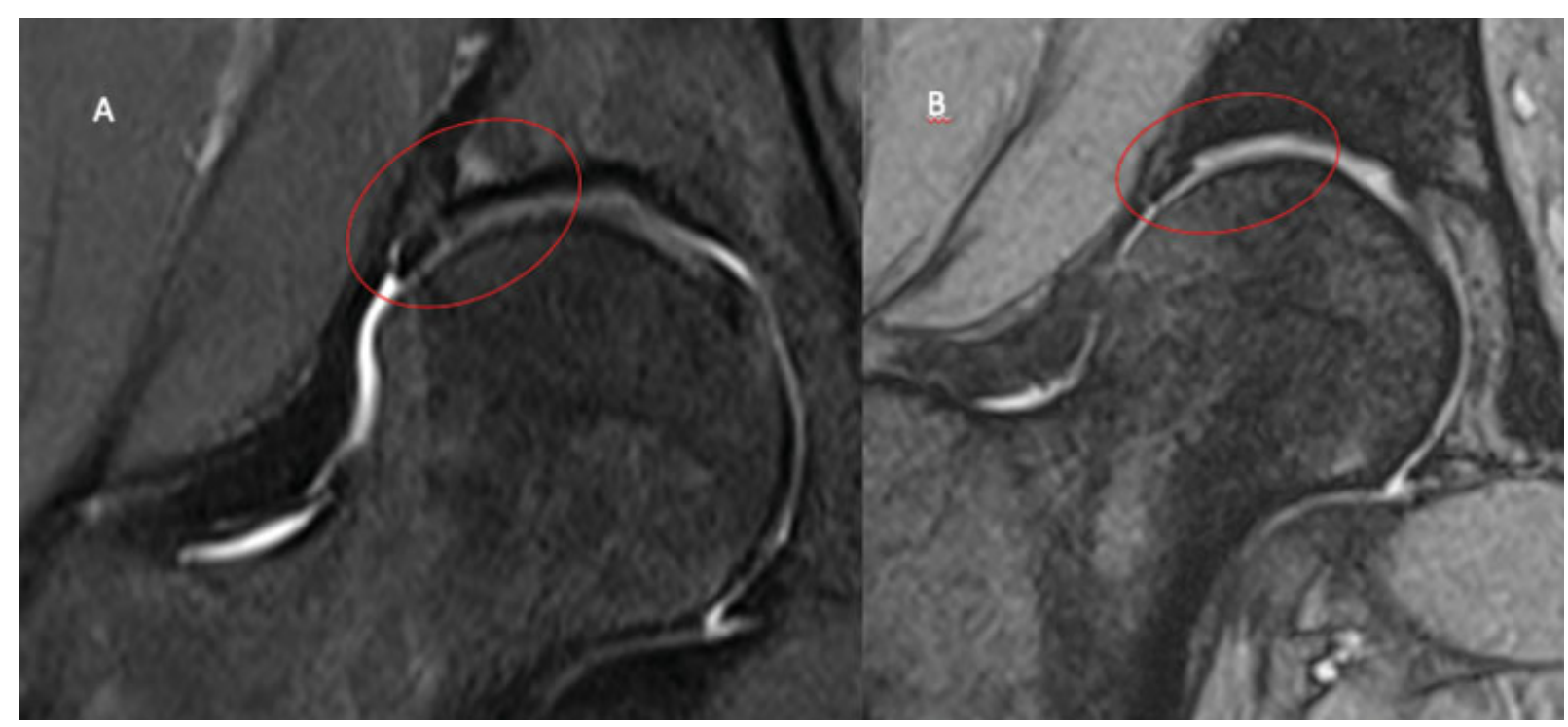

Fig. 4 (A) Imagem destacada do pré-operatória que corresponderia a lesão em onda, é possível observar a alteração da cartilagem na junção condrolabral. (B) Seis meses de pós-operatório, imagem destacada mostra que a cartilagem, onde foi feita a microfratura, apresenta o mesmo sinal e as mesmas características das áreas não lesadas. 
escura, com hipossinal nas sequencias ponderada em DP FAT SAT e gradiente-eco GRET2 em comparação com a cartilagem acetabular sadia. Na RNM após 6 meses da microfratura reversa, a área cartilaginosa tratada apresenta o mesmo sinal que a cartilagem adjacente não lesionada nos 18 pacientes avaliados, o que pode sugerir que a cartilagem está sadia e aderida ao osso subcondral, corroborando com a melhora clínica e dos escores avaliados.

A única complicação observada no presente estudo foi a formação de um cisto na região das perfurações em um paciente. Não encontramos outros estudos na literatura atual que tenha demonstrado a ocorrência desta mesma complicação. Acreditamos que tenha ocorrido a entrada de líquido sinovial pelo orifício das microfraturas com o desenvolvimento do cisto.

O ideal para confirmação de um bom resultado do procedimento seria a realização de uma nova artroscopia para biópsia do local da lesão. No entanto, isso representaria um procedimento invasivo adicional, o que envolve aspectos éticos, que, portanto, impossibilitaram sua realização.

\section{Conclusão}

O tratamento da lesão em onda através da microfratura reversa se mostrou eficaz e seguro em um seguimento de 6 meses, com melhora significativa do HHS e EVA da dor, e com achados de imagem semelhantes aos da cartilagem sadia adjacente em exames de RNM. Estudos adicionais com maior casuística e maior tempo de seguimento são necessários para definir o impacto desta nova técnica na prática clínica.

\section{Suporte Financeiro}

Não houve apoio financeiro de fontes públicas, comerciais, ou sem fins lucrativos.

\section{Conflito de Interesses}

Os autores declaram não haver conflito de interesses.

\section{Referências}

1 Wulf CA, Larson CM. Chondral Lesion. In: Byrd JW, Guanche C. AANA Advanced Arthroscopy: The Hip. Philadelphia: Elsevier; 2010:33-50

2 Chahla J, LaPrade RF, Mardones R, et al. Biological Therapies for Cartilage Lesions in the Hip: A New Horizon. Orthopedics 2016;39 (04):e715-e723

3 De Lazari LC, Laguna CB, Picado CHF, Garcia FL. Reverse Microfracture of the Hip Acetabulum: A Technique for the Wave Lesion. Arthrosc Tech 2018;7(06):e607-e610

4 Haughom BD, Erickson BJ, Rybalko D, Hellman M, Nho SJ. Arthroscopic Acetabular Microfracture With the Use of Flexible Drills: A Technique Guide. Arthrosc Tech 2014;3(04):e459-e463

5 Byrd JW. Operative Hip Arthoroscopy. 3rd ed. New Youk: Springer; 2013

6 Stone AV, Mehta N, Beck EC, et al. Comparable patient-reported outcomes in females with or without joint hypermobility after hip arthroscopy and capsular plication for femoroacetabular impingement syndrome. J Hip Preserv Surg 2019;6(01):33-40

7 Fisher RA. The logic of inductive inference. J R Stat Soc 1935;98 (01):39-82

8 Guimarães RP, Alves DP, Azuaga TL, et al. Tradução e adaptação transcultural do "Harris Hip Score modificado por Byrd". Acta Ortop Bras 2010;18(06):339-342

9 Philippon MJ, Schenker ML, Briggs KK, Maxwell RB. Can microfracture produce repair tissue in acetabular chondral defects? Arthroscopy 2008;24(01):46-50

10 Trask DJ, Keene JS. Analysis of the Current Indications for Microfracture of Chondral Lesions in the Hip Joint. Am J Sports Med 2016;44(12):3070-3076

11 MacDonald AE, Bedi A, Horner NS, et al. Indications and Outcomes for Microfracture as an Adjunct to Hip Arthroscopy for Treatment of Chondral Defects in Patients With Femoroacetabular Impingement: A Systematic Review. Arthroscopy 2016;32 (01):190-200.e2

12 Tzaveas AP, Villar RN. Arthroscopic repair of acetabular chondral delamination with fibrin adhesive. Hip Int 2010;20(01):115-119

13 Marquez-Lara A, Mannava S, Howse EA, Stone AV, Stubbs AJ. Arthroscopic Management of Hip Chondral Defects: A Systematic Review of the Literature. Arthroscopy 2016;32(07):1435-1443

14 Fontana A. Autologous Membrane Induced Chondrogenesis (AMIC) for the treatment of acetabular chondral defect. Muscles Ligaments Tendons J 2016;6(03):367-371

15 Kilıçoğlu Öİ, Polat G, Erşen A, Birişik F. Long-term result of mosaicplasty for femoral head osteochondral lesion: a case report with 8 years follow-up. Hip Int 2015;25(06):589-592 